\title{
Rimbp, a New Marker for the Nervous System of the Tunicate Ciona robusta
}

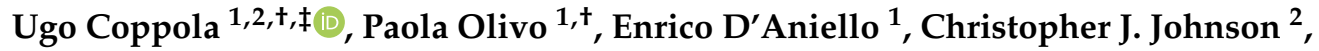 \\ Alberto Stolfi ${ }^{2, *}$ and Filomena Ristoratore ${ }^{1, *}$ \\ 1 Biology and Evolution of Marine Organisms, Stazione Zoologica Anton Dohrn, 80121 Napoli, Italy; \\ Ugo.Coppola@cchmc.org (U.C.); paola.olivo@szn.it (P.O.); enrico.daniello@szn.it (E.D.) \\ 2 School of Biological Sciences, Georgia Institute of Technology, Atlanta, GA 30332, USA; \\ cvjohnson1215@gatech.edu \\ * Correspondence: alberto.stolfi@biosci.gatech.edu (A.S.); filomena.ristoratore@szn.it (F.R.) \\ + These authors contributed equally to the work. \\ $\ddagger$ Current Address: The Heart Institute and Division of Molecular Cardiovascular Biology, Cincinnati \\ Children's Hospital Medical Center, Cincinnati, OH 45229, USA.
}

Received: 21 July 2020; Accepted: 25 August 2020; Published: 27 August 2020

\begin{abstract}
Establishment of presynaptic mechanisms by proteins that regulate neurotransmitter release in the presynaptic active zone is considered a fundamental step in animal evolution. Rab3 interacting molecule-binding proteins (Rimbps) are crucial components of the presynaptic active zone and key players in calcium homeostasis. Although Rimbp involvement in these dynamics has been described in distantly related models such as fly and human, the role of this family in most invertebrates remains obscure. To fill this gap, we defined the evolutionary history of Rimbp family in animals, from sponges to mammals. We report, for the first time, the expression of the two isoforms of the unique Rimbp family member in Ciona robusta in distinct domains of the larval nervous system. We identify intronic enhancers that are able to drive expression in different nervous system territories partially corresponding to Rimbp endogenous expression. The analysis of gene expression patterns and the identification of regulatory elements of Rimbp will positively impact our understanding of this family of genes in the context of Ciona embryogenesis.
\end{abstract}

Keywords: bipolar tail neurons; phylogeny; peripheral nervous system; CNEs; enhancers

\section{Introduction}

The exocytosis of neurotransmitter-filled synaptic vesicles of nerve and muscle cells is stabilized by various homeostatic signaling systems [1-4]. Modulation of presynaptic neurotransmitter release relies on an evolutionarily conserved form of homeostatic plasticity in neuromuscular junctions (NMJs), occurring in many distantly-related models ranging from insects to mammals $[4,5]$. The main mechanisms involved in presynaptic homeostasis are the modulation of $\mathrm{Ca}^{2+}$ influx and the regulation of the readily releasable pool of synaptic vesicles. The presynaptic active zone contains many conserved proteins such as Rab3-interacting molecules (RIMs), RIM-binding proteins (Rimbps), Munc13, ELKS's, and $\alpha$-liprins [6]. Among these, RIMs are likely to be the central organizers that mediate direct or indirect interaction with both the remaining active zone-proteins and with those contained in the synaptic vesicles [7,8]. Rimbp proteins interact with Rims1 and Rims 2 and are thus important components of the presynaptic active zone $[9,10]$ as effectors of the small GTPase Rab3, which is central to regulate the protein composition of the active zone [11-13]. Furthermore, together with voltage-gated $\mathrm{Ca}^{2+}$-channels, Rimbp and RIM proteins are thought to be fundamental in the formation of scaffolds for tethering synaptic vesicles [8]. Rimbps are implicated in the specific control of presynaptic P/Q-type 
calcium channels through Bassoon [14], confirming that these are crucial players within the presynaptic active zone and voltage-gated $\mathrm{Ca}^{2+}$ channels. During evolution, Rimbps retained the same domain organization, composed of three SH3- domains and two to three fibronectin III (FN3) repeats [15]. To date, Rimbp genes have been studied mainly in vertebrates $[9,10,15]$ with expression in the brain reported both in newborn and adult rats [15]. In mammals, mutations of RIMBP2 are associated with hearing loss [16] and ones of RIMBP3 with male infertility [17]. Concerning invertebrates, in the fly Drosophila melanogaster, a direct role of Rimbp in modulating the calcium-dependent vesicle release in the active zone $[18,19]$ has also been reported, suggesting a conserved role in synapses.

To shed light on the evolutionary history of these proteins, we performed phylogenetic and synteny analyses of Rimbp genes within animals, demonstrating that several duplications have shaped the evolution of this family. To gain insights into Rimbp expression in invertebrates, we focused our attention on the sole ortholog of Rimbp proteins present in the tunicate Ciona robusta, an invertebrate chordate considered the closest living relative of vertebrates [20]. Whole-mount in situ hybridization (WISH) experiments revealed that during Ciona embryogenesis two isoforms of Rimbp are differentially expressed in pigment cell precursors (otolith and ocellus) and in cells belonging to the Ciona peripheral nervous system (PNS). We showed that Rimbp intronic regulatory elements are able to drive expression in papilla neurons, ascending motor ganglion neurons (AMGNs), and bipolar tail neurons (BTNs). Altogether, our results highlight Rimbp expression in the nervous system of tunicates such as Ciona, paving the way to future studies on the evolution of Rimbp different roles in neurotransmitter release.

\section{Materials and Methods}

\subsection{Phylogenetic and Synteny Analysis}

The amino acid sequences used for the evolutionary survey were retrieved from the NCBI, Ensembl, ANISEED databases, and collected in Table S1, whilst the sequences characterized by a high degree of divergence were listed in Table S2. The Rimbp of tunicate Ciona robusta (XP_009858695.2) was the initial query employed for tBlastn [21], and reciprocal Blasts were performed. The obtained protein sequences were aligned using Clustal Omega [22] and the phylogenetic reconstruction was computed with the Maximum Likelihood (ML) estimation using MEGA6 with 1000 replicates and the LG substitution model $(\gamma=4$ and proportion of invariable sites $=0.4)$ [23], and all the sites were used for the phylogenetic analysis. The graphical representation was carried out with Dendroscope [24]. The syntenic survey of Rimbp genes in vertebrates was performed consulting NCBI, Ensembl, and Genomicus databases. The domain architecture of Rimbp proteins in Figure S1 was assessed by using the domain bank of PROSITE database [25].

\subsection{Animals and Embryo Electroporation}

Adults of Ciona robusta were gathered from the Gulf of Taranto or from San Diego, CA, USA, (M-REP). Gametes from several animals were taken separately for in vitro cross-fertilization followed by dechorionation and electroporation, as previously described [26-29]. Embryos were staged according to the developmental timeline established in Hotta et al. 2007 [30]. To visualize GFP, embryos were fixed in MEM-FA (3.7\% methanol-free formaldehyde, 0.1 M MOPS pH 7.4, 0.5 M NaCl, $2 \mathrm{mM}$ $\mathrm{MgSO}_{4}, 1 \mathrm{mM}$ EGTA) for $20 \mathrm{~min}$ and washed several times in PBS with $0.15 \%$ Triton X-100, $0.05 \%$ Tween 20. The transgenesis experiments were carried out electroporating 70 micrograms of construct. Each experiment was performed 4 times, and at least 200 embryos were scored.

\subsection{In Situ Hybridization}

Single in situ hybridizations were carried out essentially as illustrated previously $[27,28]$, employing DIG-labeled riboprobes in combination with anti-Dig_AP antibody (Roche, Indianapolis, IN, USA). The antisense riboprobe of the isoform A (800 bp) was PCR-amplified from cDNA of Ciona larvae (oligos listed in Table S5) and cloned into P-gem T-Easy vector (Promega, Madison, WI, USA). The antisense RNA probe was transcribed using T7 RNA Polymerase (Roche, Indianapolis, IN, 
USA) and purified with a Qiagen kit. Instead, the riboprobe of the isoform B was obtained from the Satoh library (plate: R1CiGC06d17), then linearized and transcribed employing T7 RNA Polymerase. The riboprobe of Rab3Gap1 gene was also obtained from the Satoh library (plate: R1CiGC38o19), next, linearized and transcribed using T7 RNA Polymerase.

\subsection{Molecular Cloning}

The intronic regions of Rimbp were selected by using the mVISTA tool of the ANISEED database [31] and PCR-amplified from genomic DNA (sequences are listed in Table S4). The products were cloned into a TOPO-TA vector (Invitrogen, Carlsbad, CA, USA) and then inserted into a vector comprising $e G F P$ and human $\beta$-globin minimal promoter [32]. Oligos used for cloning experiments are listed in Table S5.

\section{Results}

\subsection{Evolutionary Survey of Rimbp2/3 Genes}

In order to clarify the orthology of a gene previously named Bzrap (benzodiazepine receptor-associated protein) [28], found to be FGF-regulated in Ciona pigmented cells, corresponding to the KyotoHoya gene model KH.C5.558 [33], we performed a Maximum Likelihood (ML) phylogenetic reconstruction (Figure 1) using a manually curated database (Table S1).

In vertebrates, TSPO Associated Protein 1 or Tspoap1, also known as Rimbp1, has previously been referred to as Bzrap due to its ability to interact with the peripheral benzodiazepine receptor of mitochondria [34]. Within the tunicate-specific database ANISEED [31], reciprocal BLASTs revealed that the previously named Bzrap gene of C. robusta was closely related to various Rimbp $2 / 3$ family genes, for this reason, it has been called Rimbp Domain analysis confirmed that Ciona Rimbp protein shared the domain organization based on FN3 repeats and Src homology 3 (SH3) (Figure S1). To decipher Rimbp evolutionary history, we improved a previous analysis of Rimbp genes [15] by including in our survey proteins from Porifera up to Chordates. More specifically, we analyzed sequences belonging to the following species: the sponge Amphimedon queenslandica, the ctenophore Mnemiopsis leidyi, the cnidarian Nematostella vectensis, the brachiopod Lingula anatina, the arthropods Drosophila melanogaster (insect), Anopheles gambiae (insect) and Daphnia magna (crustacean), the nematode Caenorhabditis elegans, the mollusks Crassostrea gigas (bivalve) and Octopus bimaculoides (cephalopod), the ambulacrarians Saccoglossus kowalevskii (hemichordate) and Strongylocentrotus purpuratus (echinoderm), the tunicates Oikopleura dioica (larvacean) and Ciona robusta, Phallusia mammillata, Halocynthia aurantium (ascidians), the vertebrates Petromyzon marinus (lamprey), Callorhinchus milii (cartilaginous fish), Latimeria chalumnae (coelacanth), Lepisosteus oculatus (non-teleost actinopterygian), Danio rerio (teleost), Xenopus tropicalis (amphibian), Podarcis muralis (reptile), Gallus gallus (avian), Mus musculus and Homo sapiens (mammals) (Table S1). Sequences characterized by a high degree of evolutionary divergence have been excluded from the phylogenetic study (Table S2). We did not find any Rimbp ortholog in unicellular eukaryotes and plants. This evolutionary survey based on 48 Rimbp proteins strongly supports the existence of a single Rimbp in tunicates and other invertebrates (green box) that resulted in being orthologous to both vertebrate Rimbp2 (blue box) and Rimbp3 (orange box) (Figure 1). Among the surveyed invertebrate species, sea urchin S. purpuratus was the only one showing duplication (here, referred to as Rimbpa and Rimbpb), with Rimbpb excluded from the tree given its evolutionary divergence (Table S2). The vertebrate protein classes named Rimbp2 and Rimbp3 (Figure 1 and Table S2) encompass various proteins that in genome databases (Ensembl, NCBI) are named in a different manner (see Table S3 for old and new names). Our phylogenetic reconstruction clearly demonstrated that the human protein known as Tspoap1 belongs to Rimbp3 cluster (Figure 1), thus we renamed it as RIMBP3A. Moreover, the human gene known as RIMBP3 has been renamed RIMBP3D (Figure 1 and Table S3). This analysis allowed the identification of several Rimbp 2 and Rimbp3 duplicates in distantly related vertebrates such as zebrafish, coelacanth, and human (Figure 1 and Table S2). The existence of Rimbp duplicates 
could have resulted from isolated cases of gene duplication or from one of the two whole-genome duplications that occurred at the stem of vertebrates [35,36]. To better understand Rimbp evolution in chordates, we surveyed the conservation of the Rimbp genome environment (Figure S2), allowing us also to define the orthology of vertebrate Rimbps better. In vertebrates, Rimbp 2 genes belong to a region comprising a conserved linkage formed by Ran and Stx genes [37], which is not syntenic with Rimbp3 genomic locus (Figure S2). These findings suggest a local duplication at the root of vertebrates involving the ancestor of these two genes. Interestingly, ascidian Rimbp (C. robusta, Halocynthia aurantium) and vertebrate Rimbp3 genes preserved chromosomal vicinity with the mitochondrial E3 ligase Mul1 [38]. Furthermore, in the tunicates Ciona robusta and Phallusia mammillata, Rimbp forms a triplet (Figure S2) with Rab3Gap1, which in vertebrates is implicated in Warburg and Martsolf syndromes and, like Rimbp proteins, is an effector of Rab3 proteins [39-41], and Chmp2 [42]. In synthesis, our evolutionary data strongly support an orthology of ascidian Rimbp with the invertebrate Rimbp and with vertebrate Rimbp 2 and Rimbp3 genes, which are affected by diverse duplication events.

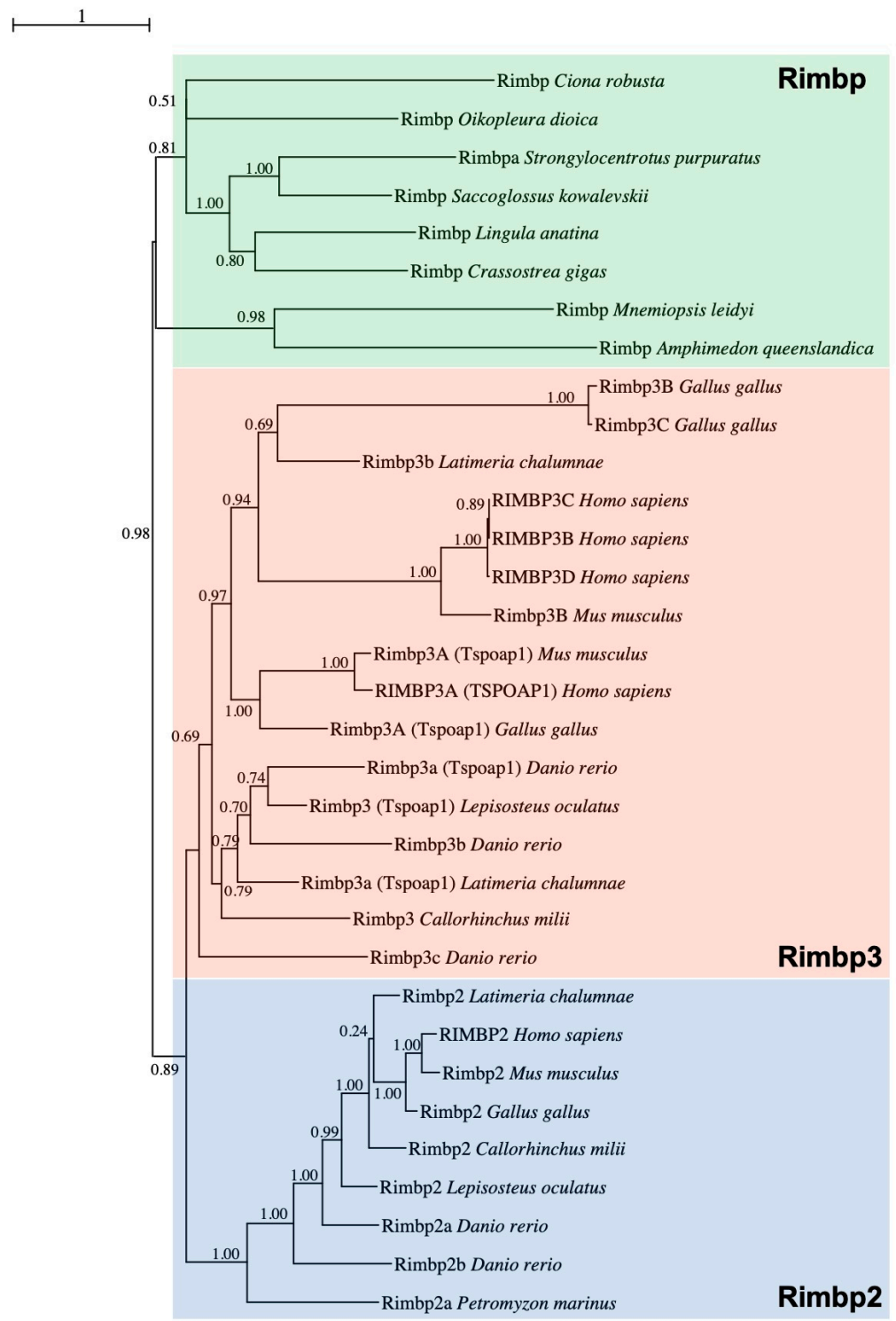

Figure 1. The evolutionary history of Rimbp proteins. Maximum Likelihood (ML) phylogenetic tree supporting the orthology among Rimbp of invertebrates (green box) and both Rimbp2 (blue box) and Rimbp3 (orange box) of vertebrates, with brackets highlighting the name commonly used for several Rimbp3. Values at branches represent replicates obtained using the ML estimation method; the whole protein sequence has been used for tree inference. 


\subsection{Dynamic Expression Pattern of Rimbp in the Ciona robusta Nervous System}

To garner an understanding of Rimbp expression in invertebrate chordates, we investigated its expression pattern during Ciona robusta embryogenesis by whole-mount in situ hybridization (Figure 2).
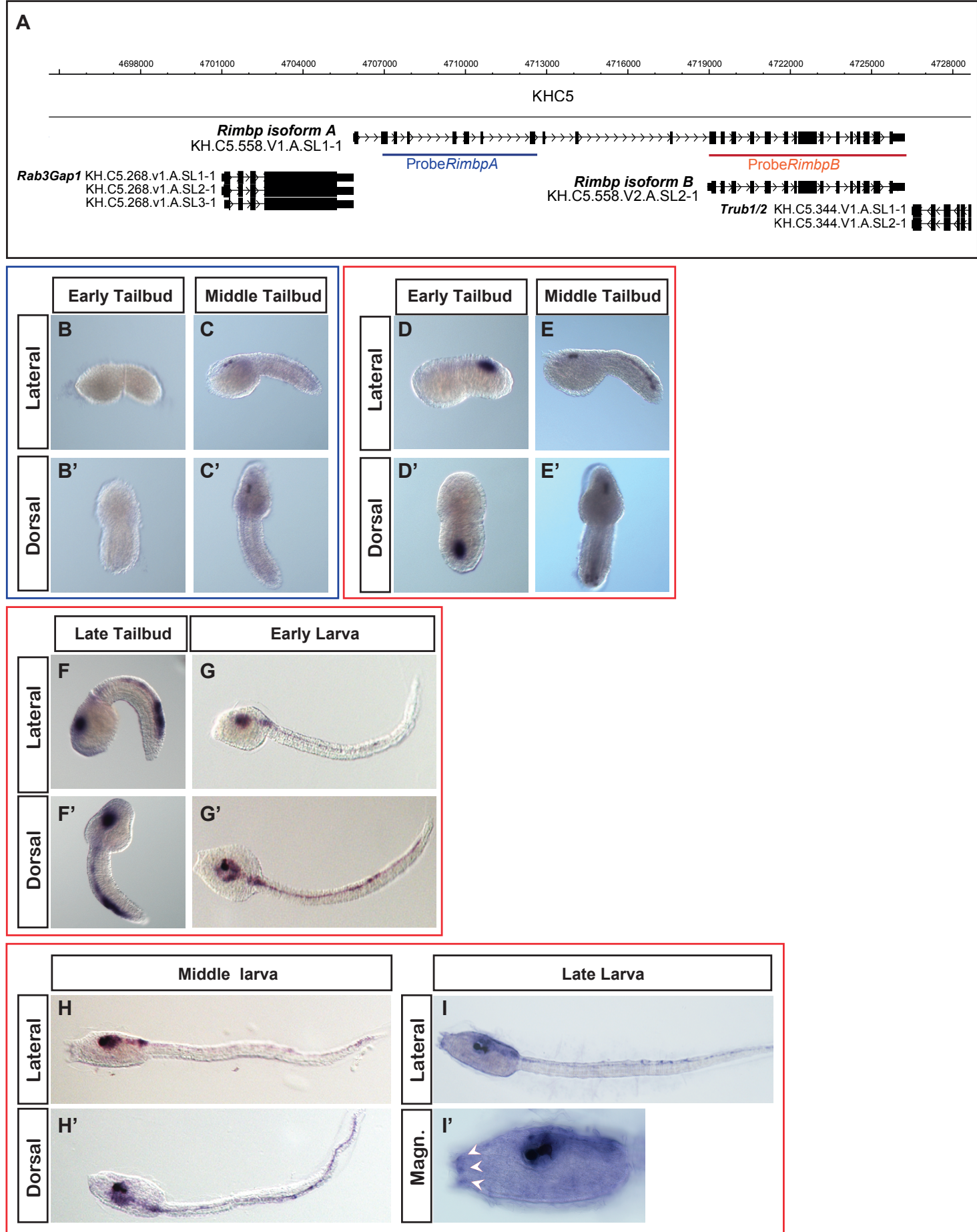

Figure 2. Ciona robusta Whole-mount in situ hybridization (WISH) of Rimbp. (A) Genomic organization of isoform A (blue) and isoform B (red) of Rimbp. (B-C'): isoform A is expressed only in the sensory vesicle at the middle tailbud stage $\left(\mathbf{C}, \mathbf{C}^{\prime}\right)$. (D-I') isoform $B$ expression. At the early tailbud stage, this isoform is expressed in the posterior part of the developing nervous system $\left(\mathbf{D}, \mathbf{D}^{\prime}\right)$ while from the middle to late tailbud, expands its expression in the sensory vesicle (E-F'). At early and middle larva stages, isoform $B$ is also expressed in the sensory vesicle and motor ganglion $\left(\mathbf{G}-\mathbf{H}^{\prime}\right)$. At the middle and late larva, the isoform $B$ starts to be expressed in the adhesive papillae organs (white arrowhead $\mathbf{H}-\mathbf{I}^{\prime}$ ). 
Our previous data showed that this gene was expressed in pigment cell precursors at the middle tailbud stage [28]. A more detailed analysis of the genomic locus using the ANISEED database showed that Ciona Rimbp exhibits two different transcript isoforms, the longer one measuring $4.7 \mathrm{~kb}$ (isoform $\mathrm{A}$ ) and the shorter that measures $3.3 \mathrm{~kb}$ (isoform B). More in details, we found that the isoform $\mathrm{B}$ does not include the first 11 exons of the isoform A (Figure 2A), although both isoforms encompass the $\mathrm{SH} 3$ and FN3 domains. Moreover, the isoform A-unique exons encode an $\mathrm{N}$-terminal protein sequence that is present in human RIMBP3 proteins, but not in RIMBP2 (Figure S1).

To distinguish between the expression of two isoforms, we synthesized two probes: one encompassing only the first region of the isoform A (blue) and specific for this isoform, the other comprising all of the isoform B (red), thus potentially able to recognize both the isoforms (Figure 2A). Isoform A was expressed exclusively at the middle tailbud stage in the sensory vesicle with specific expression in two cells corresponding to ocellus and otolith pigment cell precursors (Figure 2B- $\mathrm{C}^{\prime}$ ), as shown in our previous survey [28]. Regarding the isoform B, we found that its expression starts during the early tailbud stage in the posterior part of the developing nervous system, in what appears to be A8.16-derived ependymal cells [43] (in the lateral rows of the neural tube (Figure 2D, $\mathrm{D}^{\prime}$ ). As development proceeds, from the middle to late tailbud, we found expanded Rimbp isoform B expression in the sensory vesicle, indicating expression in larval brain neurons (Figure 2E- $\mathrm{F}^{\prime}$ ). In the posterior region, the expression also expanded to include the bipolar tail neurons (BTNs) [44], which have been compared to dorsal root ganglia neurons (DRGNs) of vertebrates [45]. Between the late tailbud and early larva, we also detected expression in the motor ganglion. At the early larva stage, the isoform $\mathrm{B}$ continued to be expressed in neurons of the brain, motor ganglion, and tail (Figure 2G, $\mathrm{G}^{\prime}$ ). Interestingly, at the middle and late larva stages, we detected expression also in the adhesive papillae organs (Figure $2 \mathrm{H}-\mathrm{I}^{\prime}$ ). Due to overlap of probe $\mathrm{B}$ with both isoforms, we cannot say if the detected expression in the pigmented cell precursors (Figure $2 \mathrm{E}, \mathrm{E}^{\prime}$ ) is specific of the isoform $\mathrm{B}$ or results from the isoform A expression.

Our characterization of Rimbp expression pattern in tunicate Ciona robusta demonstrated, for the first time, that Rimbp isoforms have differential expression patterns and activation time. Indeed, isoform B shows a strong expression in different areas of the nervous system starting from the early tailbud up to the larva stage, while isoform A is transiently expressed only in the pigment cells at the middle tailbud.

\subsection{Intronic Cis-Regulatory Elements for Rimbp Expression}

In light of Rimbp's dynamic expression in the nervous system, we focused on its regulatory logics during development (Figure 3). However, the identification of a "canonical" promoter or upstream regulatory region(s) responsible for Rimbp expression was not possible because this gene is located in close proximity to the Rab3Gap1 gene (Figure 3A) in an arrangement that suggests the formation of a putative "two-gene" operon [46].

We tried to isolate the regulatory region upstream of Rab3Gap1, but despite several attempts, we did not succeed, possibly due to problems in this genomic region. Therefore, to find regulatory regions underlying Rimbp expression, we took advantage of the mVISTA tool available on ANISEED to identify highly conserved non-coding elements (CNEs) with the sibling species Ciona savignyi within Rimbp introns (Figure 3A and Table S4). We cloned four conserved CNEs upstream of a GFP reporter gene [32], respectively, named int $4 / 5(1.8 \mathrm{~Kb})$, intR7 $(0.3 \mathrm{~Kb})$, intR11 $(0.7 \mathrm{~Kb})$, intR16 $(0.3 \mathrm{~Kb})$ (Figure 3A). Specifically, for intR7 region, we isolated two partially overlapping fragments named intR7A and intR7B (Table S4 and Figure 3A). The selected fragments were electroporated in Ciona eggs to investigate their capability to drive expression of the reporter gene in the same territory of the endogenous transcript. Results at the larval stage showed that the intR7A>eGFP, intR7B>eGFP, and intR11>eGFP were able to drive expression in some cells of the peripheral nervous system (PNS) (Figure 3B,C), possibly including BTNs, papillae neurons, and AMGs. In contrast, intR4/5>eGFP and intR16 $>e G F P$ did not show any GFP signal (Figure 3C). Both intR7A and intR7B constructs drove a strong expression in the PNS of a majority of electroporated larvae, whilst intR11>eGFP expression 
signal was reported in the PNS of only a few embryos (less than 10\%) (Figure 3C). Even though the intR7 constructs encompass overlapping regions, the intR7A>eGFP expression showed a significantly higher rate in terms of larvae expressing GFP (Figure 3C). Our analyses, however, did not reveal a strong positive signal of the reporter gene in the pigmented cell precursors, brain neurons, or ependymal cells of the tail, as shown for the endogenous transcript (Figure 2) suggesting that active motifs for this territory are not all present in the isolated regulatory elements. To better understand the intR7>eGFP expression in various neurons of the Ciona PNS at the larva stage, we analyzed, in better detail, the larvae electroporated with intR7B $>e G F P$ (Figure 4).
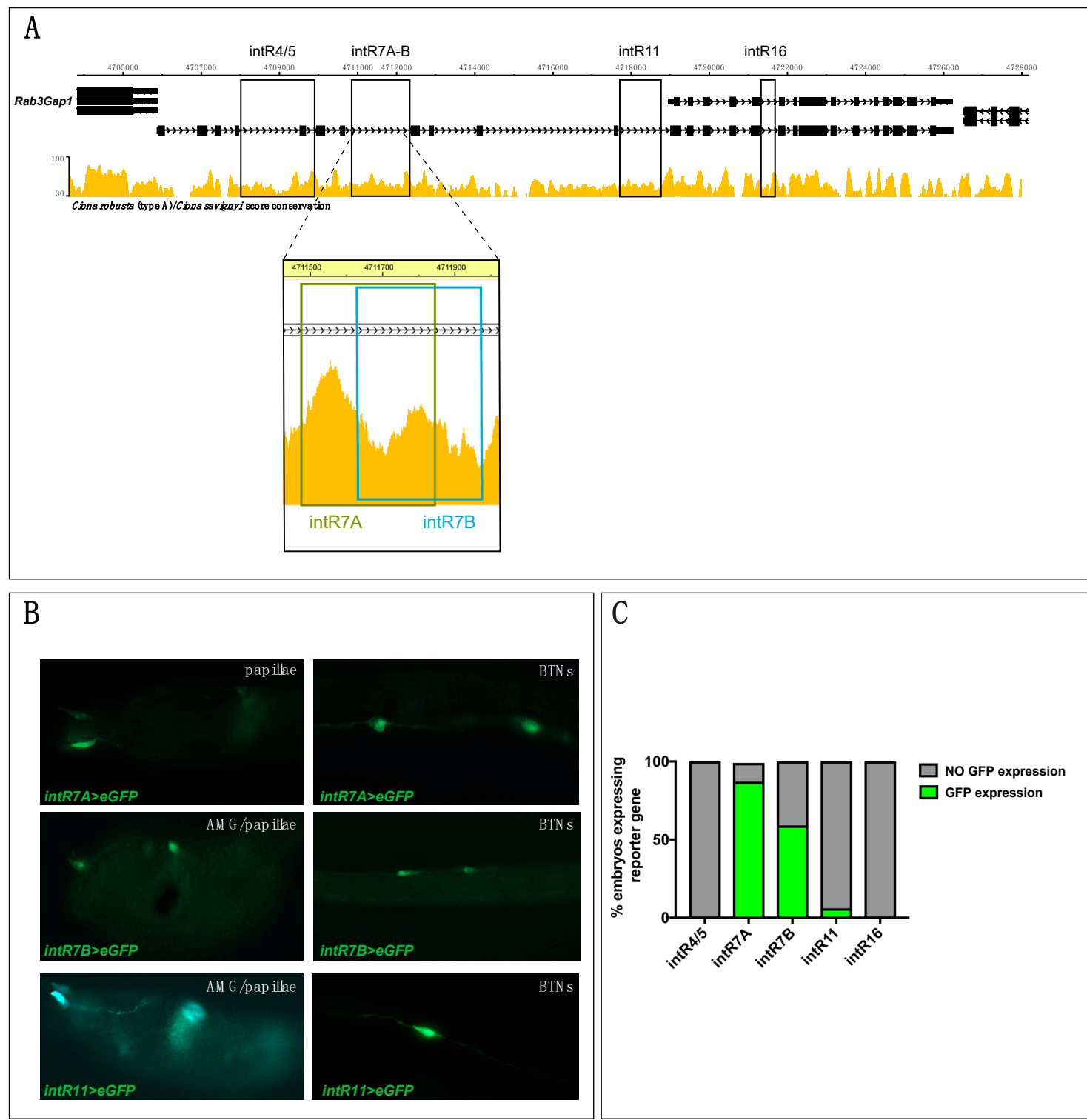

Figure 3. Intronic Cis-regulatory elements of Rimbp of Ciona robusta. (A) The genomic region comprising Rimbp and Rab3Gap1 with an automatic mVISTA plot between C. robusta and C. savignyi (ANISEED); rectangles indicate the intronic regions selected for cloning in eGFP vector (intR4/5, intR7, intR11, intR16). Dashed lines show a higher magnification of the two distinct regions selected for the intR7 (intR7A and intR7B). (B) Expression of larvae electroporated with 70 micrograms of intR7A>eGFP, intR7B $>\mathrm{eGFP}$, and intR11>eGFP in different PNS territories of larva (st. 26) possibly including BTNs, papillae neurons, and AMGNs. (C) Percentages of larvae electroporated along with intR4/5, intR7, and intR11, intR16 expressing with GFP in at least one type of cells of the nervous system. Each experiment was performed four times and at least 200 embryos were scored per each experiment. 

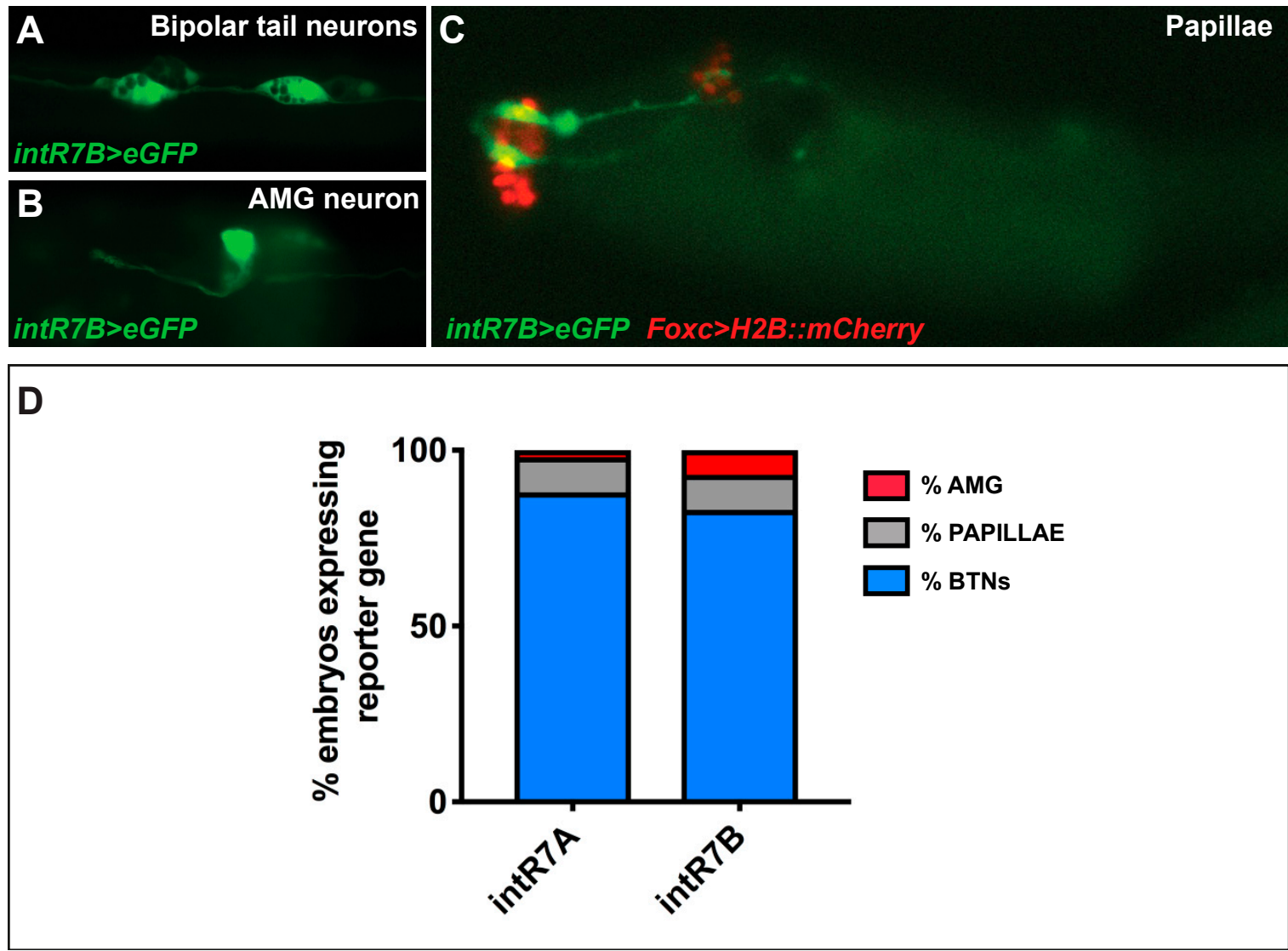

Figure 4. intR7>eGFP in PNS of Ciona robusta. (A) GFP expression in bipolar tail neurons (BTNs). (B) Expression in the neurons of ascending motor ganglion (AMG). (C) Co-expression of intR7B $>$ GFP and FoxC $>$ H2BmCherry (for both we electroporated 70 micrograms) in glutamatergic neurons of palps. (D) Percentages of larvae expressing in $t R 7 A$ and in $R 7 B$, highlighting the relative expression in distinct nervous system territories. Each experiment was performed four times, and at least 200 embryos were scored.

As for the endogenous signal, we observed a strong expression in BTNs, [44,47] (Figure 4A,D). These neurons are situated on either side of the neural tube and extend their axons along the entire length of the tail. Electroporated larvae also showed strong expression in ascending motor ganglion or AMG neurons (AMGNs), which are peripheral interneurons located dorsally to the core motor ganglion [47] (Figure 4B). The intR7 guided expression, also in neurons of the papillae, which are glutamatergic neurons that project their axons along the rostral PNS and into the sensory vesicle [48]. Papilla neuron expression of intR7 was confirmed by double electroporation along with Fox $\mathrm{C}>\mathrm{H} 2 \mathrm{BmCherry}$, which marks the papilla territory [48] (Figure 4C). Hence, we found that both intR7A and intR7B drive expression in the same nervous territories with the signal in BTN cells represented the vast majority, with $80 \%$ of positive larvae (Figure $4 \mathrm{D}$ ).

In summary, although we were not able to identify the regulatory region(s) responsible for the whole endogenous signal, we discovered three Rimbp intronic enhancers active in the PNS of Ciona robusta (intR7A, intR7B, intR11).

\section{Discussion}

Since the evolution of Rimbp genes is not well-known, we reconstructed the history of this family in animals. Rimbp proteins are considered to represent a fundamental tool for the establishment of presynaptic machinery in metazoans [49,50]: This speculation is in agreement with Rimbp absence in plants and the majority of unicellular eukaryotes but the presence in choanoflagellates (Monosiga 
brevicollis) and in non-bilaterian animals (sponges, ctenophores). The high degree of conservation of Rimbp genes in all the animals is coherent with their conserved role in presynaptic protein dynamics. Our genomic survey showed that invertebrates possess a single Rimbp gene, with the only the exception of the sea urchin exhibiting an independent duplication, whilst gnathostomes have Rimbp2 and Rimbp3 duplicates (Figure 1, Table S2 and Figure S2). The concept of orthology among invertebrate Rimbp, Rimbp2, and Rimbp3 genes is enforced by the synteny we found between human RIMBP3A and Rimbp of ascidians (Figure S2). Moreover, a strong phylogenetic signal proved that Rimbp genes are preserved in all the surveyed tunicates (Figures 1 and S2), despite these animals had undergone massive gene losses [51,52], suggesting an essential role for Rimbp.

Although the Rimbp 2 and Rimbp3 loci are conserved among gnathostomes ([37]; Figure S2), there is lack of synteny between them and in the lamprey (considered an ancestor of gnathostomes) we found only two Rimbp 2 genes (Figure 1 and Table S2). Therefore, we suggest that Rimbp 2 and Rimbp 3 orthologs derived from a gene duplication event occurred at gnathostome evolutionary radiation, as strongly suggested by the presence of a single copy of both Rimbp 2 and Rimbp3 in cartilaginous fish C. milii and in non-teleost fish L. oculatus (Figure 1). The topology of the tree and the syntenic analysis evidenced a stable evolutionary history for the Rimbp 2 lineage and major diversification for Rimbp3, with distinct origins for several vertebrate paralogs. Because the relationship existing among the various Rimbp3 duplicates was not clarified using BLAST and phylogeny, synteny analysis permitted us to define the orthology of Rimbp3 genes (Figure S2). In fact, Rimbp3a and Rimbp3b conserved in coelacanth and zebrafish do not correspond to human RIMBP3A and RIMBP3B (Figures 1 and S2): The former derives from a tandem duplication, the latter from an independent gene duplication event. Importantly, the presence of one Rimbp3 gene in amphibians, two Rimbp3 in reptiles, and three Rimbp3 genes in chicken (Figure 1 and Table S2) lead to hypothesize the existence of three duplicates in the ancestor of tetrapods (with the fourth possibly emerged in primates). Another explanation is that Rimbp3 duplication has occurred during amniote evolution. Phylogeny and synteny data speak in favor of a specific duplication event followed by a double tandem duplication with the successive loss of the fourth member. Otherwise, the presence in mouse and other mammals (dog, pig) of only two Rimbp3 genes suggests further lineage-specific losses. The additional member of zebrafish (rimbp3a2) possibly arose from teleost specific whole-genome duplication (TSGD) or 3R [53,54], as indicated by the retained synteny on chromosomes 5 and 15 (Figure S2) conserved in other teleost genomes as golden-line barbel, goldfish (NCBI, Ensembl). Moreover, the presence of Syntaxin2 (stx2) orthologs close to both zebrafish Rimbp2 gene loci (Figure S2), lead us to hypothesize the same origin for them. Among tetrapod Rimbp3 genes, the most ancient member is $\operatorname{Rimbp} 3 A$, as testified by its conserved synteny and the partial preservation with the Rimbp genome environment of ascidians (Figure S2). Moreover, if we consider the whole-genome duplications involving vertebrates, the current number of Rimbp repertoire in vertebrate models clarifies that many Rimbp orthologs have been lost. Thus, Rimbp evolution was shaped mainly by gene duplications and massive losses (in particular Rimbp3). Discovering new insights about Rimbp expression and functions in other vertebrate model systems will be important in understanding the impact of genomic rearrangements on the evolution of presynaptic functions.

In mammals, while RIMBP2 is associated with presynaptic functions $[14,55,56]$, RIMBP3 proteins seem to have a role in microtubule organization, especially in spermatozoa [17,57]. Possibly, these different biological roles can be associated with their divergent domain architecture (Figure S1). In Ciona, two different isoforms of Rimbp have been found: Both share the same domain structure (Figure S1) and the genome environment (Figure S2) with human Rimbps3 but the shorter Ciona isoform lacks the N-terminus region specific of RIMBP3 proteins. It is tempting to speculate that in invertebrate chordates Rimbp played multiple functions through alternative transcripts. Then, some ancient sub-functionalization could have been cemented genetically through the various duplications occurring in vertebrates. In light of domain organization of $N$. vectensis, the most parsimonious explanation is that the Rimbp ancestor possessed three FN3 motifs, with high degree of variability 
among metazoan Rimbps. In particular, we registered distinct FN3 losses in various species, with the dramatic case of total absence in C. milii Rimbp3.

To gain information on this family in invertebrates, we analyzed, for the first time, the expression and the regulation of the sole Rimbp in the tunicate Ciona robusta (Figures 2 and 3). In situ hybridization of Rimbp during Ciona embryogenesis revealed a substantial difference in the expression pattern between isoform A and B. The first is active only in pigment cell precursors during the early and middle tailbud stage, while it does not show any expression at the larva stages, suggesting a transient expression. The isoform B instead is expressed in cells of the central and peripheral nervous systems starting from the middle tailbud stage until the larva stages (Figure 2). Due to the fact that probe B is not able to distinguish among the two isoforms, we cannot say if the isoform B is also expressed in pigment cell precursors or not.

Regarding the regulatory mechanisms underlying Rimbp dynamic expression, we found three intronic regions (intR7A, intR7B, intR11) driving expression in neurons of the peripheral nervous system, even if with different efficiencies (Figure 3). These regions are conserved between Ciona sibling species, but no preservation with other tunicates and/or vertebrates has been observed. We did not find any regulatory element able to drive expression in the central nervous system (brain and ependymal cells of the tail) nor in the pigment cell precursors, suggesting that additional regulatory regions remain unidentified. Where the regulatory region specific for the isoform A is located remains not clear due to close proximity of another gene, Rab3Gap1, which expression is not detectable employing WISH and forming a putative operon together with Rimbp. Traditionally considered as a prokaryotic characteristic, operons have been proposed to be a specialized feature of the unusually compact Ciona genome [58]. Intriguingly, like Rimbp proteins, Rab3Gap1 is implicated in the regulation of Rab3 in the context of presynaptic dynamics [59]. Although operons in Ciona do not necessarily encompass genes with similar functions [58], the fact that both Rab3Gap1 and Rimbp proteins are functionally related in the cell, together with their chromosomal vicinity, could suggest common gene regulation modality [60,61]. Moreover, the preservation of Rab3Gap1-Rimbp duplet (Figure S2) within P. mammillata (Figure S2) and the genome conservation between C. robusta and P. mammillata (ANISEED browser) in this region, which evokes similar genomic organization in both the species.

Interestingly, the specific expression of isoform A in the cells of pigment cell lineage and its similarities with RIMBP3 organization evokes the possibility that Rimbp of Ciona has similar cellular functions of mammalian Rimbp3. It is alluring to speculate that they have a common role in the stabilization of microtubules, which could have a role in the formation of Ciona pigment cell structure. Consequently, it would be significant to gain insights on the Rimbp functions in Ciona and on the role in embryogenesis of Rimbp orthologs in other invertebrates. The expression of Rimbp in most of the Ciona larval nervous system suggests this gene encodes an important effector of neuronal function in this invertebrate chordate.

In sum, our results confirm the putatively conserved role for Rimbp proteins in neuronal presynaptic function and provide insights into the potential role of Rimbp gene duplications and subfunctionalization in the evolution of the vertebrate nervous system.

Supplementary Materials: The following are available online at http:/www.mdpi.com/2073-4425/11/9/1006/s1. Figure S1: Scheme of domain organization in metazoan Rimbp proteins; Figure S2: Comparison of genomic loci of three ascidians; Table S1: FASTA encompassing the Rimbp protein sequences used for the phylogenetic tree of Figure 1; Table S2: FASTA with the Rimbp protein sequences found using Blast and excluded from phylogeny of Figure 1 for their divergence; Table S3: List of employed Rimbp sequences, with old and new names, according to phylogenetic and syntenic data; Table S4: Intronic sequences with relative chromosomal positions that have been cloned in eGFP vector and tested via electroporation; Table S5. List of oligos used for cloning experiments.

Author Contributions: Conceptualization, U.C., E.D., A.S. and F.R.; performed experiments U.C., P.O., C.J.J.; validation, U.C., P.O., E.D., A.S. and F.R.; formal analysis, U.C., P.O., E.D., C.J.J., A.S. and F.R.; writing-original draft preparation, U.C. and P.O.; writing-review and editing, E.D., A.S. and F.R.; supervision, F.R. and A.S.; final approval of the version to be published; U.C., P.O., E.D., C.J.J., A.S. and F.R. All authors have read and agreed to the published version of the manuscript. 
Funding: U.C. has been supported by SZN OU PhD fellowship and by short-term fellowships from EMBO (7534) and The Company of Biologists (DEVTF-171108). P.O. has been supported by SZN OU PhD fellowship. A.S. is supported by NIH grant HD084814, and C.J.J. is supported by an NSF Graduate Research Fellowship.

Conflicts of Interest: The authors declare no conflict of interest.

\section{References}

1. Davis, G.W. Homeostatic control of neural activity: From phenomenology to molecular design. Annu. Rev. Neurosci. 2006, 29, 307-323. [CrossRef] [PubMed]

2. Marder, E. Variability, compensation, and modulation in neurons and circuits. Proc. Natl. Acad. Sci. USA 2011, 108 (Suppl. 3), 15542-15548. [CrossRef]

3. Turrigiano, G.G. The self-tuning neuron: Synaptic scaling of excitatory synapses. Cell 2008, 135, $422-435$. [CrossRef]

4. Davis, G.W. Homeostatic signaling and the stabilization of neural function. Neuron 2013, 80,718-728. [CrossRef]

5. Plomp, J.J.; van Kempen, G.T.; Molenaar, P.C. Adaptation of quantal content to decreased postsynaptic sensitivity at single endplates in alpha-bungarotoxin-treated rats. J. Physiol. 1992, 458, 487-499. [CrossRef] [PubMed]

6. Wojcik, S.M.; Brose, N. Regulation of membrane fusion in synaptic excitation-secretion coupling: Speed and accuracy matter. Neuron 2007, 55, 11-24. [CrossRef] [PubMed]

7. Mittelstaedt, T.; Alvaréz-Baron, E.; Schoch, S. RIM proteins and their role in synapse function. Biol. Chem. 2010, 391, 599-606. [CrossRef]

8. Kaeser, P.S.; Deng, L.; Wang, Y.; Dulubova, I.; Liu, X.; Rizo, J.; Südhof, T.C. RIM Proteins Tether Ca2 Channels to Presynaptic Active Zones via a Direct PDZ-Domain Interaction. Cell 2011, 144, 282-295. [CrossRef]

9. Wang, Y.; Sugita, S.; Sudhof, T.C. The RIM/NIM family of neuronal C2 domain proteins. Interactions with Rab3 and a new class of Src homology 3 domain proteins. J. Biol. Chem. 2000, 275, 20033-20044. [CrossRef]

10. Hibino, H.; Pironkova, R.; Onwumere, O.; Vologodskaia, M.; Hudspeth, A.J.; Lesage, F. RIM Binding Proteins (RBPs) Couple Rab3-Interacting Molecules (RIMs) to Voltage-Gated Ca2 Channels. Neuron 2002, 34, 411-423. [CrossRef]

11. Schoch, S.; Gundelfinger, E.D. Molecular organization of the presynaptic active zone. Cell Tissue Res. 2006, 326, 379-391. [CrossRef]

12. Schoch, S.; Mittelstaedt, T.; Kaeser, P.S.; Padgett, D.; Feldmann, N.; Chevaleyre, V.; Castillo, P.E.; Hammer, R.E.; Han, W.; Schmitz, F.; et al. Redundant functions of RIM1 $\alpha$ and RIM2 $\alpha$ in Ca2 -triggered neurotransmitter release. EMBO J. 2006, 25, 5852-5863. [CrossRef]

13. Graf, E.R.; Daniels, R.W.; Burgess, R.W.; Schwarz, T.L.; DiAntonio, A. Rab3 dynamically controls protein composition at active zones. Neuron 2009, 64, 663-677. [CrossRef]

14. Davydova, D.; Marini, C.; King, C.; Klueva, J.; Bischof, F.; Romorini, S.; Montenegro-Venegas, C.; Heine, M.; Schneider, R.; Schröder, M.S.; et al. Bassoon Specifically Controls Presynaptic P/Q-type Ca2 Channels via RIM-Binding Protein. Neuron 2014, 82, 181-194. [CrossRef] [PubMed]

15. Mittelstaedt, T.; Schoch, S. Structure and evolution of RIM-BP genes: Identification of a novel family member. Gene 2007, 403, 70-79. [CrossRef]

16. Girotto, G.; Vuckovic, D.; Buniello, A.; Lorente-Cánovas, B.; Lewis, M.; Gasparini, P.; Steel, K.P. Expression and replication studies to identify new candidate genes involved in normal hearing function. PLoS ONE 2014, 9, e85352. [CrossRef]

17. Okuda, H.; DeBoer, K.; O'Connor, A.E.; Merriner, D.J.; Jamsai, D.; O’Bryan, M.K. LRGUK1 is part of a multiprotein complex required for manchette function and male fertility. FASEB J. 2017, 31, 1141-1152. [CrossRef]

18. Kittel, R.J. Bruchpilot Promotes Active Zone Assembly, Ca2 Channel Clustering, and Vesicle Release. Science 2006, 312, 1051-1054. [CrossRef]

19. Liu, K.S.Y.; Siebert, M.; Mertel, S.; Knoche, E.; Wegener, S.; Wichmann, C.; Matkovic, T.; Muhammad, K.; Depner, H.; Mettke, C.; et al. RIM-Binding Protein, a Central Part of the Active Zone, Is Essential for Neurotransmitter Release. Science 2011, 334, 1565-1569. [CrossRef]

20. Delsuc, F.; Brinkmann, H.; Chourrout, D.; Philippe, H. Tunicates and not cephalochordates are the closest living relatives of vertebrates. Nature 2006, 439, 965-968. [CrossRef] 
21. Gertz, E.M.; Yu, Y.-K.; Agarwala, R.; Schäffer, A.A.; Altschul, S.F. Composition-based statistics and translated nucleotide searches: Improving the TBLASTN module of BLAST. BMC Biol. 2006, 4, 41. [CrossRef] [PubMed]

22. Sievers, F.; Wilm, A.; Dineen, D.; Gibson, T.J.; Karplus, K.; Li, W.; Lopez, R.; McWilliam, H.; Remmert, M.; Söding, J.; et al. Fast, scalable generation of high-quality protein multiple sequence alignments using Clustal Omega. Mol. Syst. Biol. 2011, 7. [CrossRef]

23. Tamura, K.; Stecher, G.; Peterson, D.; Filipski, A.; Kumar, S. MEGA6: Molecular Evolutionary Genetics Analysis version 6.0. Mol. Biol. Evol. 2013, 30, 2725-2729. [CrossRef] [PubMed]

24. Huson, D.H.; Scornavacca, C. Dendroscope 3: An interactive tool for rooted phylogenetic trees and networks. Syst. Biol. 2012, 61, 1061-1067. [CrossRef]

25. de Castro, E.; Sigrist, C.J.A.; Gattiker, A.; Bulliard, V.; Langendijk-Genevaux, P.S.; Gasteiger, E.; Bairoch, A.; Hulo, N. ScanProsite: Detection of PROSITE signature matches and ProRule-associated functional and structural residues in proteins. Nucleic Acids Res. 2006, 34, W362-W365. [CrossRef]

26. Christiaen, L.; Wagner, E.; Shi, W.; Levine, M. Isolation of Sea Squirt (Ciona) Gametes, Fertilization, Dechorionation, and Development. Cold Spring Harb. Protoc. 2009, 2009, pdb.prot5344. [CrossRef]

27. Christiaen, L.; Wagner, E.; Shi, W.; Levine, M. Whole-Mount In Situ Hybridization on Sea Squirt (Ciona intestinalis) Embryos. Cold Spring Harb. Protoc. 2009, 2009, pdb.prot5348. [CrossRef]

28. Racioppi, C.; Kamal, A.K.; Razy-Krajka, F.; Gambardella, G.; Zanetti, L.; di Bernardo, D.; Sanges, R.; Christiaen, L.A.; Ristoratore, F. Fibroblast growth factor signalling controls nervous system patterning and pigment cell formation in Ciona intestinalis. Nat. Commun. 2014, 5, 4830. [CrossRef]

29. D'Aniello, E.; Pezzotti, M.R.; Locascio, A.; Branno, M. Onecut is a direct neural-specific transcriptional activator of Rx in Ciona intestinalis. Dev. Biol. 2011, 355, 358-371. [CrossRef]

30. Hotta, K.; Mitsuhara, K.; Takahashi, H.; Inaba, K.; Oka, K.; Gojobori, T.; Ikeo, K. A web-based interactive developmental table for the ascidianCiona intestinalis, including 3D real-image embryo reconstructions: I. From fertilized egg to hatching larva. Dev. Dyn. 2007, 236, 1790-1805. [CrossRef]

31. Brozovic, M.; Dantec, C.; Dardaillon, J.; Dauga, D.; Faure, E.; Gineste, M.; Louis, A.; Naville, M.; Nitta, K.R.; Piette, J.; et al. ANISEED 2017: Extending the integrated ascidian database to the exploration and evolutionary comparison of genome-scale datasets. Nucleic Acids Res. 2018, 46, D718-D725. [CrossRef]

32. Zeller, R.W.; Weldon, D.S.; Pellatiro, M.A.; Cone, A.C. Optimized green fluorescent protein variants provide improved single cell resolution of transgene expression in ascidian embryos. Dev. Dyn. 2006, 235, $456-467$. [CrossRef]

33. Satou, Y.; Mineta, K.; Ogasawara, M.; Sasakura, Y.; Shoguchi, E.; Ueno, K.; Yamada, L.; Matsumoto, J.; Wasserscheid, J.; Dewar, K.; et al. Improved genome assembly and evidence-based global gene model set for the chordate Ciona intestinalis: New insight into intron and operon populations. Genome Biol. 2008, 9, R152. [CrossRef]

34. Galiègue, S.; Jbilo, O.; Combes, T.; Bribes, E.; Carayon, P.; Le Fur, G.; Casellas, P. Cloning and Characterization of PRAX-1. J. Biol. Chem. 1999, 274, 2938-2952. [CrossRef]

35. Abi-Rached, L.; Gilles, A.; Shiina, T.; Pontarotti, P.; Inoko, H. Evidence of en bloc duplication in vertebrate genomes. Nat. Genet. 2002, 31, 100-105. [CrossRef]

36. Dehal, P.; Boore, J.L. Two rounds of whole genome duplication in the ancestral vertebrate. PLoS Biol. 2005, 3 , e314. [CrossRef]

37. Coppola, U.; Caccavale, F.; Scelzo, M.; Holland, N.D.; Ristoratore, F.; D’Aniello, S. Ran GTPase, an eukaryotic gene novelty, is involved in amphioxus mitosis. PLoS ONE 2018, 13, e0196930. [CrossRef]

38. Li, J.; Qi, W.; Chen, G.; Feng, D.; Liu, J.; Ma, B.; Zhou, C.; Mu, C.; Zhang, W.; Chen, Q.; et al. Mitochondrial outer-membrane E3 ligase MUL1 ubiquitinates ULK1 and regulates selenite-induced mitophagy. Autophagy 2015, 11, 1216-1229. [CrossRef]

39. Aligianis, I.A.; Johnson, C.A.; Gissen, P.; Chen, D.; Hampshire, D.; Hoffmann, K.; Maina, E.N.; Morgan, N.V.; Tee, L.; Morton, J.; et al. Mutations of the catalytic subunit of RAB3GAP cause Warburg Micro syndrome. Nat. Genet. 2005, 37, 221-224. [CrossRef]

40. Aligianis, I.A.; Morgan, N.V.; Mione, M.; Johnson, C.A.; Rosser, E.; Hennekam, R.C.; Adams, G.; Trembath, R.C.; Pilz, D.T.; Stoodley, N.; et al. Mutation in Rab3 GTPase-activating protein (RAB3GAP) noncatalytic subunit in a kindred with Martsolf syndrome. Am. J. Hum. Genet. 2006, 78, 702-707. [CrossRef] 
41. Borck, G.; Wunram, H.; Steiert, A.; Volk, A.E.; Körber, F.; Roters, S.; Herkenrath, P.; Wollnik, B.; Morris-Rosendahl, D.J.; Kubisch, C. A homozygous RAB3GAP2 mutation causes Warburg Micro syndrome. Hum. Genet. 2011, 129, 45-50. [CrossRef]

42. Takahashi, Y.; He, H.; Tang, Z.; Hattori, T.; Liu, Y.; Young, M.M.; Serfass, J.M.; Chen, L.; Gebru, M.; Chen, C.; et al. An autophagy assay reveals the ESCRT-III component CHMP2A as a regulator of phagophore closure. Nat. Commun. 2018, 9. [CrossRef] [PubMed]

43. Imai, K.S.; Stolfi, A.; Levine, M.; Satou, Y. Gene regulatory networks underlying the compartmentalization of the Ciona central nervous system. Development 2009, 136, 285-293. [CrossRef]

44. Imai, J.H.; Meinertzhagen, I.A. Neurons of the ascidian larval nervous system inCiona intestinalis: II. Peripheral nervous system. J. Comp. Neurol. 2007, 501, 335-352. [CrossRef]

45. Stolfi, A.; Ryan, K.; Meinertzhagen, I.A.; Christiaen, L. Migratory neuronal progenitors arise from the neural plate borders in tunicates. Nature 2015, 527, 371-374. [CrossRef]

46. Cutter, A.D.; Agrawal, A.F. The evolutionary dynamics of operon distributions in eukaryote genomes. Genetics 2010, 185, 685-693. [CrossRef]

47. Ryan, K.; Lu, Z.; Meinertzhagen, I.A. The peripheral nervous system of the ascidian tadpole larva: Types of neurons and their synaptic networks. J. Comp. Neurol. 2018, 526, 583-608. [CrossRef]

48. Wagner, E.; Stolfi, A.; Gi Choi, Y.; Levine, M. Islet is a key determinant of ascidian palp morphogenesis. Development 2014, 141, 3084-3092. [CrossRef]

49. Conaco, C.; Bassett, D.S.; Zhou, H.; Arcila, M.L.; Degnan, S.M.; Degnan, B.M.; Kosik, K.S. Functionalization of a protosynaptic gene expression network. Proc. Natl. Acad. Sci. USA 2012, 109 (Suppl. 1), 10612-10618. [CrossRef]

50. Burkhardt, P.; Sprecher, S.G. Evolutionary origin of synapses and neurons-Bridging the gap. BioEssays 2017, 39, 1700024. [CrossRef]

51. Martí-Solans, J.; Belyaeva, O.V.; Torres-Aguila, N.P.; Kedishvili, N.Y.; Albalat, R.; Cañestro, C. Coelimination and Survival in Gene Network Evolution: Dismantling the RA-Signaling in a Chordate. Mol. Biol. Evol. 2016, 33, 2401-2416. [CrossRef] [PubMed]

52. Coppola, U.; Ristoratore, F.; Albalat, R.; D'Aniello, S. The evolutionary landscape of the Rab family in chordates. Cell. Mol. Life Sci. 2019, 76, 4117-4130. [CrossRef] [PubMed]

53. Hoegg, S.; Brinkmann, H.; Taylor, J.S.; Meyer, A. Phylogenetic timing of the fish-specific genome duplication correlates with the diversification of teleost fish. J. Mol. Evol. 2004, 59, 190-203. [CrossRef] [PubMed]

54. Kuraku, S.; Meyer, A. The evolution and maintenance of HOX gene clusters in vertebrates and the teleost-specific genome duplication. Int. J. Dev. Biol. 2009, 53, 765-773. [CrossRef]

55. Grauel, M.K.; Maglione, M.; Reddy-Alla, S.; Willmes, C.G.; Brockmann, M.M.; Trimbuch, T.; Rosenmund, T.; Pangalos, M.; Vardar, G.; Stumpf, A.; et al. RIM-binding protein 2 regulates release probability by fine-tuning calcium channel localization at murine hippocampal synapses. Proc. Natl. Acad. Sci. USA 2016, 113, 11615-11620. [CrossRef]

56. Brockmann, M.M.; Maglione, M.; Willmes, C.G.; Stumpf, A.; Bouazza, B.A.; Velasquez, L.M.; Grauel, M.K.; Beed, P.; Lehmann, M.; Gimber, N.; et al. RIM-BP2 primes synaptic vesicles via recruitment of Munc13-1 at hippocampal mossy fiber synapses. Elife 2019, 8. [CrossRef]

57. Zhou, J.; Du, Y.-R.; Qin, W.-H.; Hu, Y.-G.; Huang, Y.-N.; Bao, L.; Han, D.; Mansouri, A.; Xu, G.-L. RIM-BP3 is a manchette-associated protein essential for spermiogenesis. Development 2009, 136, 373-382. [CrossRef]

58. Zeller, R.W. Computational analysis of Ciona intestinalis operons. Integr. Comp. Biol. 2010, 50, 75-85. [CrossRef]

59. Müller, M.; Pym, E.C.G.; Tong, A.; Davis, G.W. Rab3-GAP controls the progression of synaptic homeostasis at a late stage of vesicle release. Neuron 2011, 69, 749-762. [CrossRef]

60. Coppola, U.; Annona, G.; D'Aniello, S.; Ristoratore, F. Rab32 and Rab38 genes in chordate pigmentation: An evolutionary perspective. BMC Evol. Biol. 2016, 16. [CrossRef]

61. Irimia, M.; Tena, J.J.; Alexis, M.S.; Fernandez-Minan, A.; Maeso, I.; Bogdanovic, O.; de la Calle-Mustienes, E.; Roy, S.W.; Gomez-Skarmeta, J.L.; Fraser, H.B. Extensive conservation of ancient microsynteny across metazoans due to cis-regulatory constraints. Genome Res. 2012, 22, 2356-2367. [CrossRef] [PubMed] 